\title{
The effect of the titanium butterfly implant on nasal patency and quality of life*
}

\section{S.J.A.C. van den Broek and N. van Heerbeek}

Departement of Otorhinolaryngology, Radboudumc Nijmegen, the Netherlands
Rhinology 56: 4, 364-369, 2018

https://doi.org/10.4193/Rhin.17.213

*Received for publication:

October 20, 2017

Accepted: April 3, 2018

Objective: The titanium butterfly implant seems to be a powerful technique to repair nasal valve insufficiency. However, the effect of these implants on nasal patency and quality of life has been controversial due to a lack of evidence. The purpose of this study is to evaluate the objective and subjective effect of the titanium butterfly implant on nasal patency and corresponding quality of life.

Methods: Nasal patency and quality of life of 32 patients undergoing a titanium butterfly implant were evaluated by measuring peak nasal inspiratory flow (PNIF) and completing three validated questionnaires: the NOSE, SNOT-22 and GBI, before surgery, six weeks and six months after surgery.

Results: The mean PNIF increased significant after 6 weeks, as well as after 6 months. Both the NOSE and SNOT-22 questionnaire scores showed a significant decrease at both post-operative measurements. The GBI indicated a significant increase at 6 weeks and 6 months. There were no significant differences between both of the post-operative measurements.

Conclusion: This is the first study indicating that the titanium butterfly implant provides a significant and clinically relevant increase in nasal patency and corresponding quality of life in patients with nasal valve insufficiency.

Key words: nasal surgical procedures, nasal obstruction, prostheses and implants, quality of life, nose disease

\section{Introduction}

Nasal obstruction is one of the most common symptoms seen by otorhinolaryngologists. In each individual patient there can be more than one causal factor contributing to insufficient nasal patency. Therefore diagnosis is often complex. A significant proportion of patients with nasal obstruction suffer from nasal valve insufficiency ${ }^{(1)}$. The nasal valve area is the smallest cross sectional area of the nose and contributes to about 50\% of the total airway resistance in the respiratory tract. A structural or physical alteration in the nasal valve region can lead to numerous disabling symptoms like nasal obstruction leading to disturbed sleep and fatigue. At the same time it can have a negative impact on the quality of life ${ }^{(1-5)}$.

Several surgical techniques to treat nasal valve insufficiency have been described. The cartilaginous butterfly graft has been found to be one of the most effective surgical techniques avai- lable for widening and strengthening the internal nasal valve, as well as supporting the external nasal valve ${ }^{(6-10)}$. The cartilaginous butterfly graft does, however, have some disadvantages including the loss of elasticity and strength over time, fullness of the cartilaginous nasal dorsum, and inability to adjust the graft later. In order to correct some of these limitations, Hurbis (11-13) developed a titanium-expanded polytetrafluoroethylene (PTFE) butterfly implant and demonstrated efficacy for improving nasal obstruction. Since 2003 a full titanium butterfly implant (Breathe-Implant, Heinz Kurz GmbH, Dusslingen, Germany) is available and implanted in patients with nasal valve repair. There are some essential differences with the PTFE implant developed by Hurbis. The titanium butterfly implant is placed and sutured directly on and to the cartilaginous structures of the nose, whereas the PTFE implant is placed in dissected pockets in the supraperichrondrial plain, without fixation. The approach of 


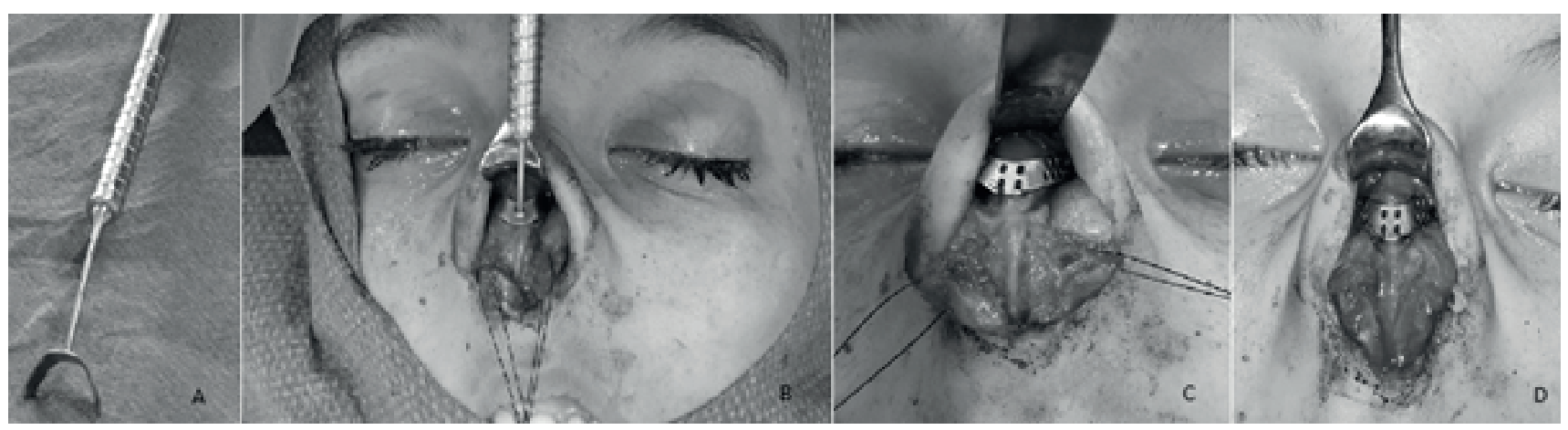

Figure 1. A) Instrument to measure size of implant. B) Placing instrument to measure size. C) Placement of implant and fixation to triangular cartilages. D) Repositioning of alar cartilage and final fixation of implant.

both techniques differs as well; the PTFE implants were placed through a vertical skin incision on the cartilaginous nasal dorsum leaving a visible scar on the nose, while the titanium butterfly implant is placed through an open rhinoplasty approach. Due to these essential differences, along with the material, both procedures are difficult to compare.

The titanium butterfly implant seems to be one of the most successful surgical techniques for patients suffering from nasal obstruction symptoms caused by nasal valve insufficiency. Although widely used in nasal valve repair, there is, however, a lack of evidence to support these claims. The studies of Hurbis (11-13) have shown promising results but a different kind of implant was studied and their outcome measurements (acoustic rhinomanometry, inspiration photographs and self-developed questionnaires) have significant limitations. Acoustic rhinomanometry only partially measures the benefit of the butterfly implant, neglecting any dynamic nasal valve collapse. Inspiration photographs register external nasal valve collapse but fail to register dynamic collapse of the internal nasal valve. The patient questionnaires used to quantify the subjective nasal obstruction were not validated or reliable to assess these symptoms and lack supporting literature.

To date, there remains a lack of compelling evidence to demonstrate the positive effects of the titanium butterfly implant. This study was performed to investigate the effect of the titanium butterfly implant on both the objective and subjective nasal patency, and the corresponding quality of life using peak nasal flow measurements and internationally accepted and validated quality of life questionnaires.

\section{Materials and methods}

All patients who underwent a titanium butterfly implant procedure in a tertiary referral hospital (Radboudumc Nijmegen, the Netherlands) since May 2014 until June 2016 were included. All patients had internal nasal valve insufficiency, sometimes combined with external nasal valve insufficiency. The indication for this procedure was stated based on patients previous history of surgery, their complaints, and physical examination. Not all of these patients were revision cases, but most of these patients had undergone prior surgery, some in our own center but often in other centers. The assessment for the indication was done by two ENT doctors specialized in facial surgery, always caring to choose the most optimal treatment for each individual patient. The indication for a titanium butterfly implant as the main treatment was always determined based on symptoms, history of previous surgery and clinical examination. For each patient the most optimal treatment was considered. A butterfly implant is considered to be a very effective device to improve nasal valve insufficiency, but has the disadvantage of possible external widening of the middle third of the nose. Other techniques to treat nasal valve insufficiency, such as spreader grafts, spanning sutures, and others may not have this disadvantage but may be less effective. In most patients any form of nasal dilator was used prior to surgery, either as an alternative to surgery or to determine whether surgery could be effective ${ }^{(14)}$. Both advantages as well as disadvantages of different techniques were discussed with each patient, after which the best strategy was chosen. All patients were seen and operated on by one of the centers two ENT doctors specialized in nasal surgery. Possible confounding parameters such as prior rhinoplasty, the use of nasal spray, age, the size of the implant, and the surgeon performing the procedure were documented.

\section{Study design}

All patients were included pre-operatively and followed at least six months after surgery. In order to determine an objective value of nasal patency at baseline, the peak nasal inspiratory flow (PNIF) of each patient was measured. Chaves et al. show that the PNIF is a reliable tool to objectively measure nasal patency ${ }^{(15)}$. To measure the subjective value of nasal obstruction and corresponding quality of life, three questionnaires were used: the NOSE; the SNOT-22; and the GBI. All of these have previously proven to be reliable tools in subjectively determining nasal patency in patients ${ }^{(16)}$. Baseline measurements consisted of the 
Table 1. Patient characteristics.

\begin{tabular}{|lcc|}
\hline \multicolumn{1}{|c}{ Characteristic } & Total (percentage \%) \\
\hline Gender & Male & $23(71.9)$ \\
\hline Size butterfly graft & Female & $9(28.1)$ \\
& L & $7(21.9)$ \\
\hline Prior surgery & XXL & $10(31.3)$ \\
\hline & Not documented & $7(21.9)$ \\
\hline Yes & $8(25)$ \\
\hline Daily nose spray & No & $26(81.3)$ \\
& Unknown & $4(12.5)$ \\
\hline & No & $3(6.3)$ \\
\hline & Yes & $29(90.6)$ \\
\hline
\end{tabular}

PNIF, the NOSE and the SNOT-22 questionnaire, which were repeated at 6 weeks and 6 months after surgery. At both of these post-operative moments the GBI questionnaire was included as well. During each follow-up visit all patients were asked for any disturbing cosmetic changes after surgery. There were no objective measurements performed.

\section{Surgical technique}

All procedures were done under general anesthesia. Through an open rhinoplasty approach the alar cartilage and cartilaginous nasal dorsum were exposed. With the use of sizer instruments the appropriate size of the titanium butterfly implant was determined (Figure 1). A size wider than the actual width of the cartilaginous nasal dorsum was chosen, such that the triangular cartilages could be sutured outward to the implant. The implant was placed and sutured onto the cartilaginous nasal dorsum and both triangular cartilages with prolene 5-0. The cephalic border of the lateral crus was positioned over the implant. Both the marginal incision and the midcolumellar incision were closed with resorbable sutures (monocryl 6-0). All patients were treated with antibiotics during and extending 1 week after the procedure.

\section{Statistical analysis}

After testing for a Gaussian distribution, within-subject analysis was performed to compare changes in the objective and subjective parameters after six weeks and six months. Confounding or effect modulating factors like size of the implant, prior surgery, gender and age were explored.

\section{Results}

In total, 32 patients (mean age $45.9 \pm 11.5$ years) were included. Thirty patients had prior nasal surgery (93.8\%), ranging from septoplasty and turbinate reduction to different rhinoplasty

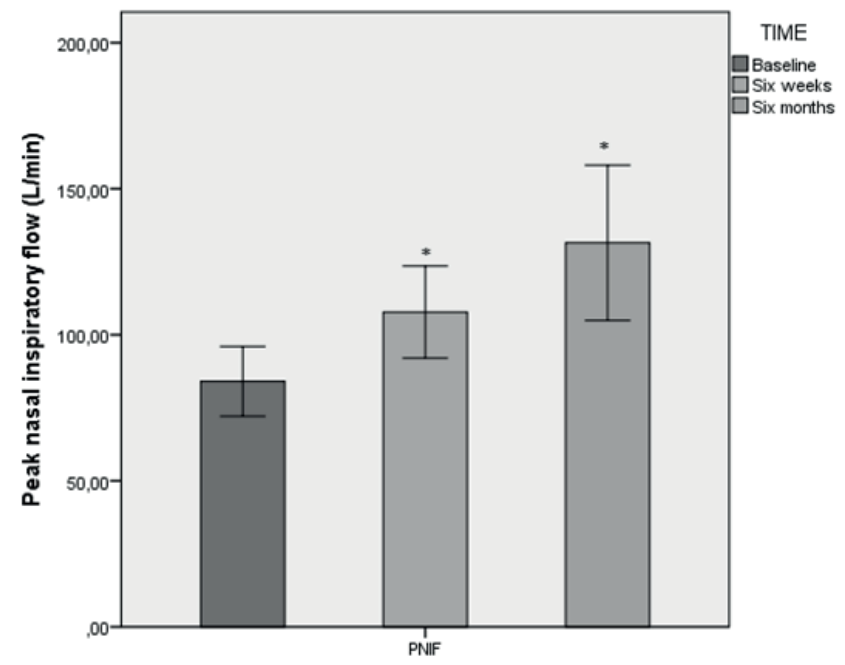

Figure 2. Mean PNIF scores at baseline, after 6 weeks and 6 months. (* Significant increase compared to baseline $(p<0.001)$.

procedures to improve nasal patency. From all patients 29 out of $32(90.6 \%)$ were using a corticoid nasal spray daily at the time of selection for the procedure. Four of the 32 patients underwent concurrent septoplasty in this study due to deviation of the septum. An overview of patient characteristics is shown in table 1.

All results are summarized in Table 2 and Figures 2 and 3. At 6 weeks a significant increase of $30.0 \mathrm{~L} / \mathrm{min}(95 \% \mathrm{Cl} 19-39.9$; $\mathrm{p}=0.000$ ) in the mean PNIF was measured, when compared to baseline. After six months the mean PNIF was showed an even further increase of $53.4 \mathrm{~L} / \mathrm{min}(95 \% \mathrm{Cl} 28.7-80.0 ; \mathrm{p}=0.000)$ above the baseline. Compared to the first postoperative measurement, an increase of $20.1 \mathrm{~L} / \mathrm{min}(95 \% \mathrm{Cl} 2.4-37.9 ; \mathrm{p}=0.029)$ was seen. After 6 weeks, the baseline mean NOSE scores were decreased from 74.5 to 42.3 , a mean decrease of 32.3 (95\%Cl -44.9- -19.6; $\mathrm{p}=0.000$ ). After 6 months, the mean NOSE score was decreased with 48.6 points $(95 \% \mathrm{Cl}-60.6--36.5 ; \mathrm{p}=0.000)$ when compared to baseline. No significant difference was found between both postoperative measurements.

The corresponding mean total SNOT-22 scores were lowered from 40.9 to 27.6 , a mean decrease of 13.3 points $(95 \% \mathrm{Cl}-17.9$ - -8.7; $\mathrm{p}=0.000)$ after six weeks. The mean SNOT-22 score was decreased by 23.7 points $(95 \% \mathrm{Cl}-34.5--12.8 ; \mathrm{p}=0.000)$ after six months, compared to baseline. No statistical differences were found between the postoperative measurements.

After 6 weeks the GBI showed a significant improvement in health status showing a mean total GBI score of $14.9(95 \% \mathrm{Cl}$ 9.9$19.8 ; p=0.000)$. When divided into three specific subscales of the $\mathrm{GBI}$, a mean difference of 19.4 points $(95 \% \mathrm{Cl} 13.2-25.5 ; \mathrm{p}=0.000)$ for the General GBI was seen, whereas the Social GBI showed a mean difference of $8.3(95 \% \mathrm{Cl} 0.96-15.7 ; \mathrm{p}=0.029)$. There was no significant change found in the Physical GBI subscale. After six 
Table 2. Mean difference with 95\% Cl of PNIF, NOSE, SNOT-22 and GBI, 6 weeks and 6 months post-operative.

\begin{tabular}{|c|c|c|c|c|c|}
\hline & & \multirow[t]{2}{*}{ Mean difference } & \multicolumn{2}{|c|}{$95 \%$ Confidence Interval } & \multirow[t]{2}{*}{ P-value } \\
\hline & & & Lower & Upper & \\
\hline \multirow[t]{3}{*}{ PNIF (L/min) } & 6 weeks -baseline & 30.0 & 19.9 & 40.0 & 0.000 \\
\hline & 6 months -baseline & 53.4 & 28.7 & 78.0 & 0.000 \\
\hline & 6 months -6 weeks & 20.1 & 2.4 & 37.9 & 0.029 \\
\hline \multirow[t]{3}{*}{ NOSE total } & 6 weeks - baseline & -32.3 & -44.9 & -19.6 & 0.000 \\
\hline & 6 months -baseline & -48.6 & -60.8 & -36.5 & 0.000 \\
\hline & 6 months- 6 weeks & -8.1 & -18.8 & 2.6 & 0.126 \\
\hline \multirow[t]{3}{*}{ SNOT-22 total } & 6 weeks -baseline & -13.3 & -17.9 & -8.7 & 0.000 \\
\hline & 6 months -baseline & -23.7 & -34.5 & -12.8 & 0.000 \\
\hline & 6 months -6 weeks & -7.1 & -17.0 & 2.8 & 0.149 \\
\hline \multirow[t]{2}{*}{ GBI Total } & 6 weeks & 14.7 & 9.9 & 19.8 & 0.000 \\
\hline & 6 months & 24.2 & 12.2 & 36.2 & 0.001 \\
\hline \multirow[t]{2}{*}{ GBI General } & 6 weeks & 19.4 & 13.2 & 25.5 & 0.000 \\
\hline & 6 months & 28.7 & 15.9 & 41.5 & 0.000 \\
\hline \multirow[t]{2}{*}{ GBI Social } & 6 weeks & 8.3 & 0.96 & 15.7 & 0.029 \\
\hline & 6 months & 5.6 & -6.1 & 17.3 & 0.331 \\
\hline \multirow[t]{2}{*}{ GBI Physical } & 6 weeks & 3.3 & -1.47 & 8.1 & 0.163 \\
\hline & 6 months & 25 & 7.4 & 42.6 & 0.008 \\
\hline
\end{tabular}

months the mean total GBI score showed a significant improvement of 24.2 points $(95 \% \mathrm{Cl} 12.2-36.2 ; \mathrm{p}=0.001)$. A mean difference of 28.7 points $(95 \% \mathrm{Cl} 15.9-41.5 ; \mathrm{p}=0.000)$ was observed for the General GBI subscale. There was no significant difference on the Social GBI subscale after six months. In contrast to the mean Physical GBI score at six weeks, the mean score at six months showed an significant improvement of 25 points $(95 \% \mathrm{Cl}$ 7.4-42.6; $p=0.014$ ).

No significant differences in outcome between the three measurement moments were found between patients who underwent concurrent surgery and patients who solely underwent implantation of the titanium butterfly.

The median total follow up was 14 months and is still ongoing. During this period there were no complications reported in the study. None of the patients suffered from wound infection or implant extrusion. None of the 32 patients reported any disturbing aesthetic changes of the nose.

\section{Discussion}

The purpose of this study was to determine the objective and subjective effect of the titanium butterfly implant on nasal patency and quality of life. This prospective cohort study followed 32 patients who were treated with a titanium butterfly implant. Three questionnaires were combined with peak nasal inspiratory flow measurements to quantify the effect of this procedure. Previous evidence has shown that the PNIF corre- lates with subjective nasal patency and nasal obstruction ${ }^{(17,18)}$. Both the NOSE and SNOT-22 questionnaires are validated and reliable instruments to evaluate nasal obstruction and surgical outcome of rhinoplasty procedures ${ }^{(19-21)}$. The GBI questionnaire was specifically designed and validated to evaluate the effect of ENT interventions on quality of life ${ }^{(21,23)}$. Thus, the combination of these four measurements provides a reliable estimate of the effect on nasal patency and quality of life. The results in this study show a significant improvement on both the objective (PNIF) and subjective (questionnaires) nasal patency and quality of life, for both the short as well as the medium-long term. This is the first study to present the effects of the titanium butterfly implant with reliable and validated outcome measurements. The clinical relevance of these improvements is difficult to interpret. Overall, all of the outcome measurements, including one objective and three subjective, show a statistically significant improvement on both nasal patency and quality of life. There can be no other conclusion than that the effect of the titanium butterfly implant should be considered clinically relevant. There is minimal literature on the clinical interpretability of the outcome measurements used in this study. In a systematic review of Rhee et al. (22), on the effectiveness of the NOSE scale for surgery outcome, evidence showed that no studies reported a lower pre- to post-surgery drop of 30 points. The authors therefore stated that a 30 point drop is clinically relevant. The mean difference in NOSE scores in this study both exceed this amount, decreasing with 32.3 points and 48.6, respectively, at 6 weeks 


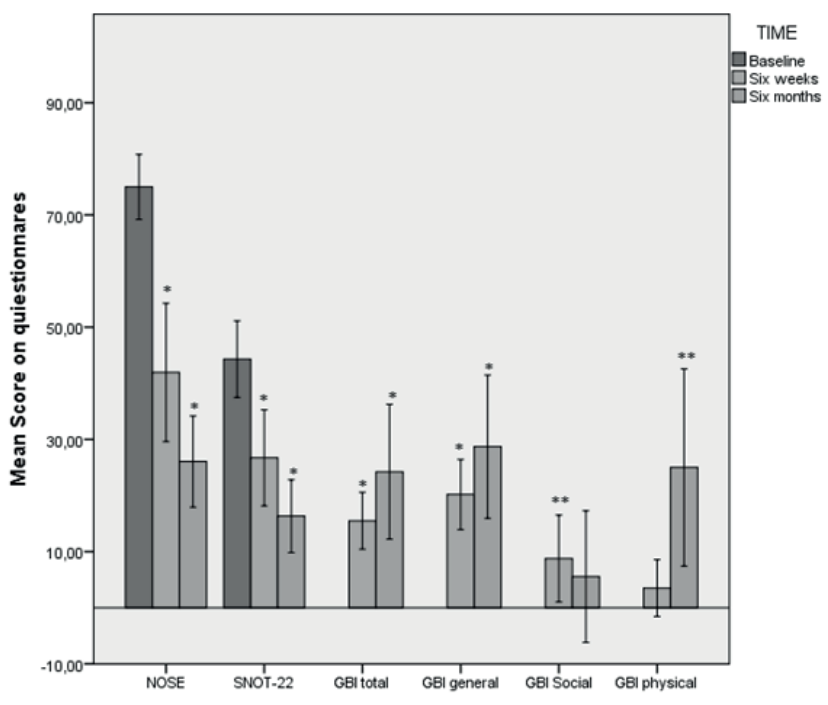

Table 3. Mean scores on NOSE, SNOT-22, and GBI questionairre at baseline, after 6 weeks and 6 months. * Significant increase compared to baseline $(p \leq 0.001) ; *$ * Significant increase compared to baseline $(\mathrm{p} \leq 0.005)$.

and 6 months. Thus, it can be concluded that the decrease in NOSE score found in this study should be considered a clinical relevant decrease.

Others have tried to calculate a Minimally Clinical Important Difference (MCID) for the SNOT-22 questionnaire, resulting in a MCID of 9 points ${ }^{(25,26)}$. In this study the mean difference in SNOT22 scores were -13.3 and -23.7 respectively for the 6 weeks and 6 months measurement. Therefore, both of these changes should be considered a clinically relevant. Timperley et al. ${ }^{(27)}$ tried to do the same for the PNIF, showing a MCID of $18 \mathrm{~L} / \mathrm{min}$ to be clinically relevant. Mean differences in this study in peak nasal inspiratory flow were $30.0 \mathrm{~L} / \mathrm{min}$ and $53.4 \mathrm{~L} / \mathrm{min}$, respectively after 6 weeks and 6 months. These changes indicate a clinical relevant improvement in peak nasal inspiratory flow.

Based on its construct, every positive score on the GBI questionnaire can be considered clinically relevant. All but two of the GBI subscale showed a significant positive difference in GBI scores in this study. Although the literature on clinical interpretability is limited there are different studies that try to determine an objective measure to determine clinical important differences, as seen above. When comparing the results found in this study, all but two of the GBI subscale should be considered clinical relevant changes. Thus, the results in this study show that the titanium butterfly implant has both a significant and an obvious clinically relevant effect on the objective and subjective nasal patency and quality of life in patients with nasal valve insufficiency. In addition, there were no complications or cosmetic side effects documented. Although in this study cosmetic outcome was not measured but only asked for at each follow-up visit, none of the patients reported any disturbing cosmetic changes of the nose after surgery. The titanium butterfly implant may nevertheless cause minor disturbances of the middle third of the nose. Others showed that the cartilaginous butterfly graft causes slight changes of the nasal width ${ }^{(28)}$. However, the titanium butterfly implant is thinner than the cartilaginous graft. In addition, all patients were informed prior to surgery about this possible side effect of the titanium butterfly implant. This may explain why none of our patients reported any disturbing cosmetic alteration of the nose.

In this study, only a small number of patients were included, which hampers the ability to perform advanced statistical analysis. Nonetheless, significant effects were found on both the short as the long term when compared to baseline. Only the peak nasal inspiratory flow showed a significant increase between 6 weeks and 6 months. However, the results of the NOSE, SNOT-22 and $\mathrm{GBI}$ at six months seem to show a trend towards an even more positive effect of the procedure compared with the results at six weeks. Future research is needed to determine the long term results of the titanium butterfly implant.

In this study 26 out of 32 patients had prior surgery. The large number of patients with prior surgery can be explained. Nasal valve insufficiency is a complicated diagnosis and is often overlooked. This frequently leads to other diagnoses and thus surgical interventions, which often have no or insufficient effect. At the same time, nasal valve insufficiency can be a complication of prior rhinoplasty. Based on this the titanium butterfly implant often is the only remaining option for many of the patients seen at our tertiary referral hospital. However, we certainly believe that the titanium butterfly implant can also be very effective for primary cases with nasal valve insufficiency. Additionally, previous studies showed that the cartilaginous butterfly graft is an excellent procedure in both primary and secondary rhinoplasty, suggesting that the titanium butterfly implant should also be applicable in both of these patient groups ${ }^{(6-8)}$.

The collective results of this study strengthen the evidence for the effectiveness of the titanium butterfly implant. There is however no literature that compares the titanium with the cartilaginous butterfly graft. None of the studies on the cartilaginous graft have used the same validated questionnaires or similar outcome measures, preventing the possibility for comparison of both methods. However, the drawbacks of the cartilaginous butterfly graft, such as loss of elasticity and strength or the inability to adjust the graft later, all favor the titanium implant. Additionally, in both this study and Hurbis' studies, there were no to minimal side effects reported ${ }^{(11-13)}$. This combined evidence appears to favor the titanium butterfly implant over the cartilaginous graft as a superior procedure for nasal valve repair. The procedure provides clinically relevant effects, has multiple benefits over the cartilaginous graft, and with limited or no side effects. However, research comparing both procedures should 
be conducted to confirm this suggestion.

\section{Conclusion}

In summary, this is the first study to evaluate the effects of the titanium butterfly implant. The findings presented in this study show both a significant as well as a clinically relevant objective and subjective improvement of nasal patency and related quality of life after placement of a titanium butterfly implant in patients with nasal valve insufficiency. There were no adverse events or aesthetic complaints reported by the patients. The titanium butterfly implant is one of the most effective surgical tools for nasal valve repair in patients suffering from nasal valve insufficiency.

\section{Acknowledgement}

None

\section{Authorship contribution}

SvdB: primray author, data collection and analysis

$\mathrm{NvH}$ : data collection, supervising author, proofreading

\section{Conflict of interest}

The authors report no conflict of interest. Ethical approval: none. Funding: none

\section{References}

1. Constantian MB. Differing characteristics in 100 consecutive secondary rhinoplasty patients following closed versus open surgical approaches. Plast Reconstr Surg. 2002;109(6):2097-111.

2. Kasperbauer JL, Kern EB. Nasal valve physiology. Implications in nasal surgery. Otolaryngol Clin North Am. 1987;20(4):699719.

3. Constantian MB, Clardy $\mathrm{RB}$. The relative importance of septal and nasal valvular surgery in correcting airway obstruction in primary and secondary rhinoplasty. Plast Reconstr Surg. 1996;98(1):38-54; discussion 5-8.

4. 4.Wittkopf M, Wittkopf J, Ries WR. The diagnosis and treatment of nasal valve collapse. Curr Opin Otolaryngol Head Neck Surg. 2008;16(1):10-3.

5. Saleh AM, Younes A, Friedman O. Cosmetics and function: quality-of-life changes after rhinoplasty surgery. Laryngoscope. 2012;122(2):254-9.

6. Clark JM, Cook TA. The 'butterfly' graft in functional secondary rhinoplasty. Laryngoscope. 2002;112(11):1917-25.

7. Andre RF, Vuyk HD. The "butterfly graft" as a treatment for internal nasal valve incompetence. Plast Reconstr Surg. 2008;122(2):73e4 e.

8. Friedman O, Cook TA. Conchal cartilage butterfly graft in primary functional rhinoplasty. Laryngoscope. 2009;119(2):255-62.

9. Chaiet SR, Marcus BC. Nasal tip volume analysis after butterfly graft. Ann Plast Surg. 2014;72(1):9-12

10. Friedman O, Coblens O. The Concha Cartilage Butterfly Graft. Facial plastic surgery: FPS. 2016;32(1):42-8

11. Hurbis CG. An adjustable, butterfly-design, titanium-expanded polytetrafluoroethylene implant for nasal valve dysfunction: a pilot study. Archives of facial plastic surgery. 2006;8(2):98-104.

12. Hurbis CG. A follow-up study of the Monarch adjustable implant for correction of nasal valve dysfunction. Arch Facial Plast Surg. 2008;10(2):142-3.

13. Hurbis CG. An adjustable implant for nasa valve dysfunction: a 3-year experience. Ear Nose Throat J. 2012 Aug;91(8):E5-12

14. Hellings PW, Nolst Trenite GJ. Improvement of nasal breathing and patient satisfaction by the endonasal dilator Airmax ${ }^{\circledR}$ Rhinology. 2014 Mar;52(1):31-4

15. Chaves C, de Andrade CR, Ibiapina C Objective measures for functional diagnostic of the upper airways: practical aspects. Rhinology. 2014 Jun;52(2):99-103.

16. van Egmond MMHT, van Heerbeek N, Ter Haar ELM, Rovers MM. Clinimetric properties of the Glasgow Health Status Inventory, Glasgow Benefit Inventory Peak Nasal Inspiratory Flow, and 4-Phase Rhinomanometry in adults with nasa obstruction. Rhinology. 2017 Jun 1;55(2):126-134

17. Fairley JW, Durham LH, Ell SR. Correlation of subjective sensation of nasal patency with nasal inspiratory peak flow rate. Clin Otolaryngol Allied Sci. 1993;18(1):19-22.

18. Ottaviano G, Scadding GK, Coles S, Lund VJ. Peak nasal inspiratory flow; normal range in adult population. Rhinology. 2006;44(1):325.

19. Buckland JR, Thomas S, Harries PG. Can the Sino-nasal Outcome Test (SNOT-22) be used as a reliable outcome measure for successful septal surgery? Clin Otolaryngol Allied Sci. 2003;28(1):43-7

20. Stewart MG, Witsell DL, Smith $T L$, Weaver EM, Yueh B, Hannley MT. Development and validation of the Nasa Obstruction Symptom Evaluation (NOSE) scale. Otolaryngol Head Neck Surg. 2004;130(2):157-63.

21. Erdogan BA, Avseren E, Paksoy M, Bora F Altin G. Assessing quality of life in septorhinoplasty patients with two different instruments. B-ENT. 2013;9(4):277-83.

22. Rhee JS, Sullivan CD, Frank DO, Kimbell JS Garcia GJ. A systematic review of patientreported nasal obstruction scores: defin- ing normative and symptomatic ranges in surgical patients. JAMA Facial Plast Surg. 2014;16(3):219-25; quiz 32.

23. Manickavasagam J, Wong S, Varabei V Raghavan U. Nasal valve surgery: assessment of quality of life with the Glasgow Benefit Inventory. Ear, Nose Throat J. 93(45):174-6.

24. Robinson K, Gatehouse S, Browning GG. Measuring patient benefit from otorhinolaryngological surgery and therapy. Ann Otol Rhinol Laryngol. 1996 Jun;105(6):41522

25. Hopkins C, Gillett S, Slack R, Lund VJ, Browne JP. Psychometric validity of the 22-item Sinonasal Outcome Test. Clin Otolaryngol. 2009 Oct;34(5):447-54.

26. Rudmik L, Soler ZM, Mace JC, DeConde AS, Schlosser RJ, Smith TL. Using preoperative SNOT-22 score to inform patient decision for Endoscopic sinus surgery. Laryngoscope. 2015;125(7):1517-22.

27. Timperley D, Srubisky A, Stow N, Marcells GN, Harvey RJ. Minimal clinically important differences in nasal peak inspiratory flow. Rhinology. 2011;49(1):37-40.

28. Chaiet SR, Marcus BC. Nasal tip volume analysis after butterfly graft. Ann Plast Surg. 2014 Jan;72(1):9-12.

S.J.A.C. van den Broek, MSc Hatertseweg 164 6533AT Nijmegen

Netherlands

Tel: +31-6-1310 6933

E-mail: Steefvdbroek@gmail.com 